\title{
Cluster-transfer reactions with radioactive beams: A spectroscopic tool for neutron-rich nuclei
}

S. Bottoni, ${ }^{1,2,3,}{ }^{*}$ S. Leoni, ${ }^{1,2, \dagger}$ B. Fornal, ${ }^{4}$ R. Raabe,${ }^{3}$ K. Rusek, ${ }^{5}$ G. Benzoni, ${ }^{2}$ A. Bracco, ${ }^{1,2}$ F. C. L. Crespi, ${ }^{1,2}$ A. I. Morales, ${ }^{2}$ P. Bednarczyk, ${ }^{4}$ N. Cieplicka-Oryńczak, ${ }^{2,4}$ W. Królas, ${ }^{4}$ A. Maj, ${ }^{4}$ B. Szpak, ${ }^{4}$ M. Callens,${ }^{3}$ J. Bouma, ${ }^{3}$ J. Elseviers, ${ }^{3}$ H. De Witte, ${ }^{3}$ F. Flavigny, ${ }^{3,6}$ R. Orlandi, ${ }^{3,7}$ P. Reiter, ${ }^{8}$ M. Seidlitz, ${ }^{8}$ N. Warr, ${ }^{8}$ B. Siebeck, ${ }^{8}$ S. Hellgartner, ${ }^{9}$ D. Mücher, ${ }^{9}$ J. Pakarinen, ${ }^{10}$ M. Vermeulen, ${ }^{11}$ C. Bauer, ${ }^{12}$ G. Georgiev, ${ }^{13}$ R. V. F. Janssens, ${ }^{14}$ D. Balabanski, ${ }^{15}$ M. Sferrazza, ${ }^{16}$ M. Kowalska, ${ }^{17}$ E. Rapisarda, ${ }^{17}$ D. Voulot, ${ }^{17}$ M. Lozano Benito, ${ }^{17}$ and F. Wenander ${ }^{17}$

${ }^{1}$ Università degli Studi di Milano, I-20133 Milano, Italy

${ }^{2}$ INFN sezione di Milano, I-20133 Milano, Italy

${ }^{3}$ KU Leuven, Instituut voor Kern-en Stralingsfysica, B-3001 Leuven, Belgium

${ }^{4}$ Institute of Nuclear Physics Polish Academy of Sciences, PL-31-342 Kraków, Poland

${ }^{5}$ Heavy Ion Laboratory, University of Warsaw, PL-02-093 Warsaw, Poland

${ }^{6}$ Institut de Physique Nucléaire d'Orsay, CNRS/IN2P3, Université Paris-Sud 11, F-91406 Orsay, France

${ }^{7}$ Advanced Science Research Center, Japan Atomic Energy Agency, Tokai, Ibaraki 319-1195, Japan

${ }^{8}$ Institut für Kernphysik der Universität zu Köln, D-50937 Köln, Germany

${ }^{9}$ Department of Physik, Technische Universität München, D-85748 Garching, Germany

${ }^{10}$ University of Jyväskylä, FIN-40014 Jyväskylä, Finland

${ }^{11}$ Nuclear Physics Group, Department of Physics, University of York, UK-YO10 5DD York, United Kingdom

${ }^{12}$ Institut für Kernphysik, Technische Universität Darmstadt, D-64289 Darmstadt, Germany

${ }^{13}$ CSNSM, CNRS/IN2P3, Universite Paris-Sud 11, UMR8609, F-91405 ORSAY-Campus, France

${ }^{14}$ Physics Division, Argonne National Laboratory, Argonne, Illinois 60439, USA

${ }^{15}$ ELI-NP, Hora Hulubei National Institute of Physics and Nuclear Engineering, 077125 Magurele, Romania

${ }^{16}$ Département de Physique, Université libre de Bruxelles, B-1050 Bruxelles, Belgium

${ }^{17}$ CERN 1211, CH-Geneva 23, Switzerland

(Received 17 June 2015; published 27 August 2015)

\begin{abstract}
An exploratory experiment performed at REX-ISOLDE to investigate cluster-transfer reactions with radioactive beams in inverse kinematics is presented. The aim of the experiment was to test the potential of cluster-transfer reactions at the Coulomb barrier as a mechanism to explore the structure of exotic neutron-rich nuclei. The reactions ${ }^{7} \mathrm{Li}\left({ }^{98} \mathrm{Rb}, \alpha \mathrm{xn}\right)$ and ${ }^{7} \mathrm{Li}\left({ }^{98} \mathrm{Rb}\right.$, txn $)$ were studied through particle- $\gamma$ coincidence measurements, and the results are presented in terms of the observed excitation energies and spins. Moreover, the reaction mechanism is qualitatively discussed as a transfer of a clusterlike particle within a distorted-wave Born approximation framework. The results indicate that cluster-transfer reactions can be described well as a direct process and that they can be an efficient method to investigate the structure of neutron-rich nuclei at medium-high excitation energies and spins.
\end{abstract}

DOI: 10.1103/PhysRevC.92.024322

PACS number(s): $25.70 . \mathrm{Hi}, 29.30 . \mathrm{Kv}, 21.10 . \mathrm{Re}, 27.60 .+\mathrm{j}$

\section{INTRODUCTION}

At present, there is a rather general consensus that a deeper understanding of nuclear structure and processes can be achieved from a focus on phenomena occurring in unexplored regions of the nuclear landscape. This view is partly based on the assumption that new observations in exotic parts of the nuclear chart will provide severe and crucial tests of the existing theoretical descriptions [1-4].

\footnotetext{
*Present address: Physics Division, Argonne National Laboratory, Argonne, IL 60439, USA.

†Corresponding author: silvia.leoni@mi.infn.it

Published by the American Physical Society under the terms of the Creative Commons Attribution 3.0 License. Further distribution of this work must maintain attribution to the author(s) and the published article's title, journal citation, and DOI.
}

In this context, high-spin yrast states in nuclei far from the valley of stability are of special interest as such excitations often arise from the maximum spin coupling of the valence particles and holes. Their wave functions usually involve mostly one well-defined configuration and are, thus, rather pure (see Ref. [5] and references therein).

A successful method for investigating the high-spin yrast structure of exotic neutron-rich nuclei uses deep-inelastic reactions occurring during heavy-ion collisions at beam energies around $20 \%$ above the Coulomb barrier. However, in such processes, the production yield is spread over many nuclei, resulting in a rather low intensity for individual species [6-17].

A more efficient technique to populate yrast states in neutron-rich nuclei is provided by cluster-transfer reactions. For example, reactions induced by a ${ }^{7} \mathrm{Li}$ beam have been exploited extensively for $\gamma$-ray spectroscopy studies of states of relatively high angular momentum in neutron-rich nuclei which are inaccessible with standard fusion-evaporation reactions involving stable beam-target combinations [18-23]. Indeed, with its pronounced alpha $(\alpha)$-triton $(\mathrm{t})$ cluster structure 
characterized by a separation energy of only $2.467 \mathrm{MeV}$ [24], ${ }^{7} \mathrm{Li}$ becomes a projectile of choice for $\alpha$ - and t-transfer reactions [25]. In such a process, it is possible to populate states with a significantly higher angular momentum than can be reached even with ${ }^{7} \mathrm{Li}$-induced fusion [18].

It is natural to anticipate that $\mathrm{t}$ transfer induced by neutronrich radioactive beams on a ${ }^{7} \mathrm{Li}$ target will populate yrast states in nuclei in the immediate vicinity of the projectile, herewith opening to yrast spectroscopy as yet unexplored parts of the nuclear chart. However, such reactions have not yet been studied and, consequently, data are needed to inform on their feasibility as well as on reaction dynamics when neutron-rich nuclei are involved.

In this paper, cluster-transfer reactions induced by a neutron-rich radioactive beam on a ${ }^{7} \mathrm{Li}$ target were investigated for the first time in a pilot experiment performed at REXISOLDE [26,27]. A ${ }^{98} \mathrm{Rb}$ beam bombarded a ${ }^{7} \mathrm{Li}$ target at a beam energy close to the Coulomb barrier. Particular attention was devoted to $\gamma$-ray spectroscopy of t- and $\alpha$-transfer products. In addition, some aspects of reaction dynamics were also investigated by confronting experimental data with theoretical calculations performed with the FRESCO code [28] using a distorted-wave Born approximation (DWBA) approach. This test experiment is meant as a first step of a broader experimental program aimed at studying the structure of yrast states in neutron-rich nuclei, in particular around doubly-magic ${ }^{132} \mathrm{Sn}$, by using cluster transfer with a ${ }^{7} \mathrm{Li}$ target.

The paper is organized as follows. In Sec. II, the ${ }^{98} \mathrm{Rb}+{ }^{7} \mathrm{Li}$ experiment is presented along with the apparatus. In Sec. III, the reaction channels observed in the present measurements are briefly described, whereas Sec. IV focuses on the clustertransfer reaction channels of interest in terms of $\gamma$ spectroscopy and of reaction dynamics aspects. Conclusions are given in Sec. V.

\section{THE EXPERIMENT}

The experiment was performed at REX-ISOLDE [26,27]. A radioactive neutron-rich beam of ${ }^{98} \mathrm{Rb}$ at a nominal energy of $2.85 \mathrm{MeV} /$ nucleon with an average intensity of $2 \times 10^{4}$ particles per second was sent onto a $1.5-\mathrm{mg} / \mathrm{cm}^{2}$-thick ${ }^{7} \mathrm{Li}$ enriched LiF target. Due to energy loss, the beam energy at midtarget was $2.49 \mathrm{MeV} /$ nucleon. Together with the ${ }^{98} \mathrm{Rb}$ ions, a strong ${ }^{98} \mathrm{Sr}$ isobaric component was observed in the beam. This ${ }^{98} \mathrm{Sr}$ contaminant originates from the limited mass separation and $\beta$ decay of ${ }^{98} \mathrm{Rb}$ during the bunching and charge-breeding phases [29]. The experimental setup consisted of the HpGe MINIBALL array [30] coupled to the Si detector system T-REX [31], and $\gamma$-particle coincidence events were measured. The MINIBALL spectrometer is a high-resolution HpGe detector array consisting of 24 sixfold segmented crystals, characterized by good spatial and energy resolutions. In the present experiment, it had a photopeak efficiency of $5 \%$ at $1 \mathrm{MeV}$. T-REX is a $\mathrm{Si}$ detector setup optimized for transfer reactions in inverse kinematics, designed to be used with the MINIBALL array. It consists of two compact disk (CD) detectors [32] placed at forward and backward angles plus eight lateral square detectors forming a barrel. In the present experiment, only the forward CD detector was used in a new configuration where it was placed at $22 \mathrm{~mm}$ from the target. This arrangement resulted in a wide $21^{\circ}-62^{\circ}$ angular coverage in the laboratory frame of reference. The forward CD detector consists of two layers with 140 and $1500-\mu \mathrm{m}$ respective thicknesses, used as a $\Delta E-E$ telescope in order to detect and identify light charged particles. The first layer is segmented into 16 annular rings and 24 vertical strips, enabling determination of the scattering angles.

The proposed reaction has two distinct features that greatly facilitate detection of the discrete $\gamma$ rays and their identification. First, the very inverse kinematics guarantees that the product nuclei all travel downstream in a small recoil cone; thus, Doppler reconstruction of the $\gamma$-ray data does not require recoil detection. Second, the reaction channels of interest are uniquely associated with the emission of an $\alpha(\mathrm{t})$ particle. By detecting the latter, one should be able to produce a very clean trigger for $\mathrm{t}(\alpha)$-transfer processes.

\section{REACTION CHANNELS}

It is well known that in heavy-ion collisions many reaction channels are available with intensities depending on several parameters, such as the relative energies, mass, charge, and $Q$-value matching conditions [33]. In particular, at energies around the Coulomb barrier, "fast" direct reactions compete with "slow" fusion-evaporation ones, and the interplay between these processes can modify significantly the balance between the cross sections for the outgoing channels. In direct reactions, transfer is one of the main processes removing flux from elastic scattering, and the coupling between different channels is a key ingredient to understand both the reaction dynamics and the microscopic structure of the interacting nuclei [9]. Furthermore, the usual couplings between different reaction channels may be altered when weakly-bound neutronrich nuclei are used as reaction partners [34]. In this section, the reaction channels observed in the present experiment with the help of particle- $\gamma$ and $\gamma-\gamma$ coincidence techniques are presented.

Figure 1 shows charged particles detected by the CD detector and identified with the $\Delta E-E_{\mathrm{T}}$ technique, where $E_{\mathrm{T}}$ is the total measured energy. The most intense channel corresponds to ${ }^{7} \mathrm{Li}$ elastic and inelastic scatterings with the former being the main component. The elastic channel is primarily associated with Rutherford scattering with a small interference due to the nuclear potential at larger angles as will be shown later. A small part of these events corresponds to the inelastic excitation of both beam components ${ }^{98} \mathrm{Rb}$ and ${ }^{98} \mathrm{Sr}$ - the associated Doppler-corrected $\gamma$ spectrum is presented in Fig. 2. It is worth noting that both Coulomb and nuclear interactions participate in projectile excitation as the beam energy exceeds the Coulomb barrier. The $2^{+} \rightarrow 0^{+}$ transition at $144 \mathrm{keV}$ in ${ }^{98} \mathrm{Sr}$ can be clearly seen together with three other strong $\gamma$ lines at 51, 95, and $115 \mathrm{keV}$. These transitions might possibly belong to the ${ }^{98} \mathrm{Rb}$ decay scheme, which is not known. The $\gamma$ rays at 51 and $115 \mathrm{keV}$ are not in coincidence with each other nor with the 144-keV line. It is very likely that some of these three $\gamma$ rays feed the ground state of ${ }^{98} \mathrm{Rb}$, whereas the other(s) feed(s) the 139-ms isomer at $270 \mathrm{keV}$ in this nucleus - the ${ }^{98} \mathrm{Rb}$ projectiles were delivered 


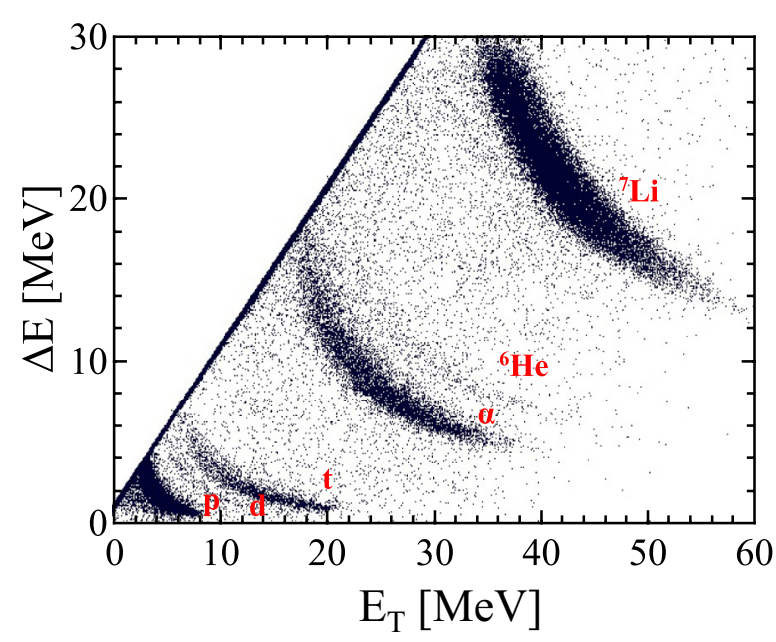

FIG. 1. (Color online) $\Delta E-E_{\mathrm{T}}$ spectrum from the CD detector enabling the identification of the following charged particles: ${ }^{7} \mathrm{Li}$ from elastic and inelastic scatterings, ${ }^{6} \mathrm{He}$ from one-proton stripping, $\alpha$ particles from $\mathrm{t}$ transfer as well as ${ }^{7} \mathrm{Li}$ and ${ }^{6} \mathrm{Li}$ breakup, tritons from $\alpha$ transfer and ${ }^{7} \mathrm{Li}$ breakup, deuterons (d) from ${ }^{6} \mathrm{Li}$ breakup, and protons ( $p$ ) from elastic scattering on target contaminants. The diagonal corresponds to particles which did not punch through the $\Delta E$ detector, such as elastically and inelastically scattered ${ }^{19} \mathrm{~F}$ nuclei.

by ISOLDE in both the ground and the isomeric states [35]. The transitions discussed above could be displayed with higher statistics by requiring a coincidence with particles with mass 7 and higher, that did not punch through the $\Delta E$ detector (diagonal of Fig. 1), as shown in the inset of Fig. 2.

This enhancement in the statistics arises from the presence in the gate of inelastically scattered low-energy ${ }^{7} \mathrm{Li}$ nuclei as well as of ${ }^{19} \mathrm{~F}$ reaction partners from the target contaminant

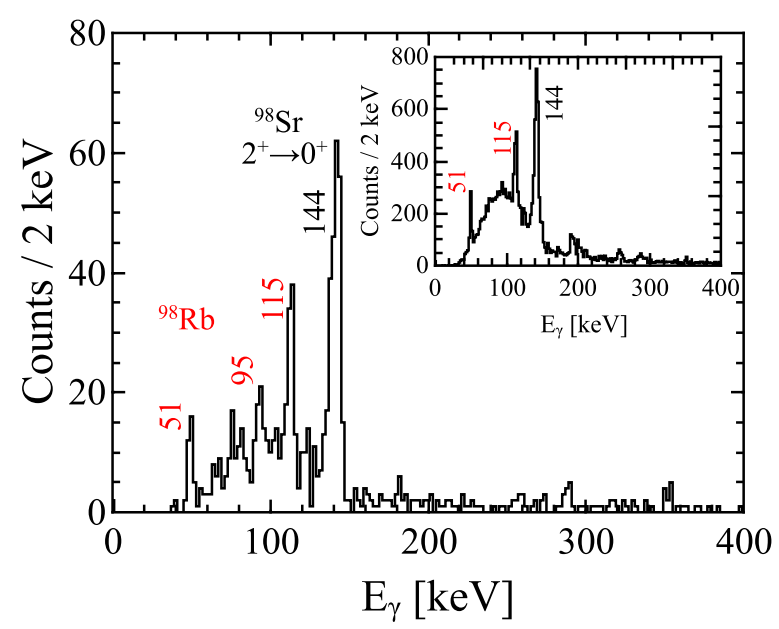

FIG. 2. (Color online) Doppler-corrected $\gamma$-ray spectra measured in coincidence with identified ${ }^{7} \mathrm{Li}$ ions showing inelastic excitations of both ${ }^{98} \mathrm{Rb}$ and ${ }^{98} \mathrm{Sr}$ beam components. While the 144-keV line is the $2^{+} \rightarrow 0^{+}$transition of ${ }^{98} \mathrm{Sr}$, the $51-, 95-$, and $115-\mathrm{keV} \gamma$ rays probably belong to ${ }^{98} \mathrm{Rb}$ (see discussion in the text). Inset: $\gamma$ rays in coincidence with all detected particles with mass 7 or higher, showing the background which arises mostly from the inclusion of scattered ${ }^{19} \mathrm{~F}$ products which did not punch through the $\Delta E$ detector. for which the inelastic cross section is larger. It must be acknowledged that the $115-\mathrm{keV}$ line was tentatively assigned to ${ }^{98} \mathrm{Rb}$ in a fission study [36]. In addition, the 51-, 95-, and $115-\mathrm{keV}$ transitions reported in this paper were recently observed at ISOLDE in a Coulomb excitation measurement induced by a ${ }^{98} \mathrm{Sr}$ beam in which ${ }^{98} \mathrm{Rb}$ was present as a contaminant [37]. It is, thus, very likely that these transitions belong to ${ }^{98} \mathrm{Rb}$.

Among events in which a Li product was identified, some could correspond to the $1 n$-stripping channel leading to ${ }^{6} \mathrm{Li}$ as this ion cannot be disentangled from ${ }^{7} \mathrm{Li}$ in the present measurement. However, the cross section for such a process is estimated to be on the order of a few millibarn only as it is hindered by a rather negative $Q$ value and does not appear to give an appreciable contribution to the spectra of Fig. 2 nor to the angular distributions of the elastically scattered ${ }^{7} \mathrm{Li}$ nuclei. One-proton stripping, leading to ${ }^{6} \mathrm{He}$, is also expected, but again, with a low cross section of few millibarn as confirmed in Fig. 1. In this case, the $Q$ value for the transfer to the ground state $Q_{\mathrm{gg}}$ is positive and rather large when compared to an expected negative optimum $Q$ value $Q_{\text {opt }}$ (see Sec. IV for the definition of $Q_{\text {opt }}$ ).

Despite the low intensity of the beam, charged particles are clearly identified, and their spectra are characterized by sizable statistics. Most of the $\alpha$ and $t$ particles detected correspond to $t$ and $\alpha$ transfers, the features of which are discussed in Sec. IV. A fraction of their yield originates, however, directly from ${ }^{7} \mathrm{Li}$ elastic breakup-this contribution was established to be less than $20 \%$ by comparing single-particle spectra and those measured in coincidence with $\gamma$ rays. A small percentage of the $\alpha$ particles comes from ${ }^{6} \mathrm{Li}$ breakup into $\alpha$ and deuteron (d). The contribution of this channel can be inferred from the presence of $\mathrm{d}$ particles as ${ }^{6} \mathrm{Li}$ produced by $1 n$ stripping can break up if its excitation energy is above $1.47 \mathrm{MeV}$.

Finally, elastic scattering on protons (these particles are target contaminants) was observed. No other channel associated with the emission of charged particles could be identified. In particular, there is no evidence for $1 p$ pickup leading to ${ }^{8} \mathrm{Be}$, which subsequently would break up into two $\alpha$ particles. Such a process was found to be quite strong in reactions studied in Ref. [38] involving stable nuclei. For neutron-rich systems where $Q_{\mathrm{gg}}$ is heavily reduced in magnitude while $Q_{\mathrm{opt}}$ is still expected to be large and positive, the $1 p$-pickup channel is hindered. This can be viewed as an example of the evolution of reaction dynamics when going towards the neutron drip line.

In the ${ }^{98} \mathrm{Rb}+{ }^{7} \mathrm{Li}$ reaction under investigation, besides direct channels, fusion-evaporation processes occur with a rather large cross section. The compound nucleus ${ }^{105} \mathrm{Zr}^{*}$ is produced at an excitation energy of $\approx 40 \mathrm{MeV}$ with a cross section on the order of $200 \mathrm{mb}$, according to predictions by the CASCADE code [39]. The same calculations indicate that $5 n$ evaporation is the main channel ( $80 \%$ of the total yield) leading to ${ }^{100} \mathrm{Zr}$ evaporation residues. Production of other $\mathrm{Zr}$ residues $\left({ }^{98,99} \mathrm{Zr},{ }^{101} \mathrm{Zr}\right)$ is also expected but with lower probability. By applying the $\gamma-\gamma$ coincidence technique and a proper Doppler correction, $\gamma$ rays from a given evaporation residue can be identified. In Fig. 3, the projection on a single axis of the prompt (200 ns) $\gamma-\gamma$ matrix is plotted. The red spectrum 


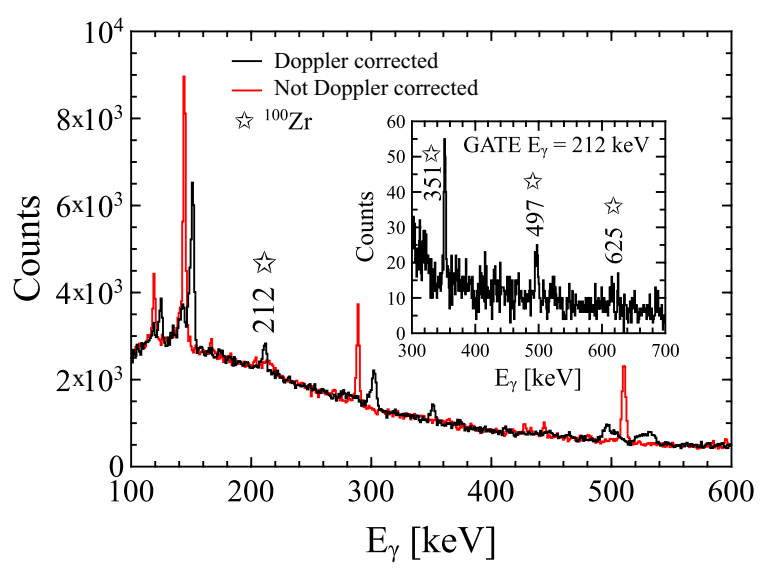

FIG. 3. (Color online) Projection of the prompt $(\approx 200 \mathrm{~ns}) \gamma-\gamma$ coincidence matrix with and without Doppler correction (black and red lines, respectively). In the Doppler-corrected spectrum, the $212-\mathrm{keV} 2^{+} \rightarrow 0^{+}$transition of ${ }^{100} \mathrm{Zr}$ can be seen, corresponding to $5 n$ evaporation. Inset: projection of the $\gamma-\gamma$ coincidence matrix gated on the 212-keV line showing the relevant coincidence relationships between yrast transitions of ${ }^{100} \mathrm{Zr}$ (labeled by stars). The last observed $\gamma$ ray at $625 \mathrm{keV}$ corresponds to the $8^{+} \rightarrow 6^{+}$transition. All other strong transitions in the spectra were identified but are not labeled for clarity.

contains events without Doppler-shift correction, whereas the black one is Doppler corrected using an average recoil velocity of $v / c \approx 0.075$. The $2^{+} \rightarrow 0^{+}$transition at $212 \mathrm{keV}$ in ${ }^{100} \mathrm{Zr}$ can be clearly seen and, in coincidence with it, the yrast band up to $8 \hbar$ as shown in the inset of the same figure. These results demonstrate that $\gamma$-ray spectroscopy of some neutron-rich nuclei can be performed by using fusion-evaporation reactions induced by radioactive beams of low intensity. In the present measurement aimed at studying products of $\mathrm{t}$ and $\alpha$ transfers, $\gamma$ rays from evaporation residues do not represent an unwanted hindrance as the evaporation residues are neutron rich and do not emit charged particles with sizable cross sections.

\section{CLUSTER TRANSFERS}

The aim of the present paper is to investigate in detail tand $\alpha$-transfer channels at the Coulomb barrier for reactions with a neutron-rich radioactive beam in inverse kinematics. These channels can be observed when a weakly-bound nucleus with a prominent cluster structure, such as ${ }^{7} \mathrm{Li}$ in this experiment, is involved. This process is favored at energies around the Coulomb barrier, below a critical value of the angular momentum associated with the relative motion of the reaction partners such that the final system can survive against fission $[21,40]$. The transferred angular momentum is rather large $(\approx$ tens of $\hbar$ ), compared to single-nucleon transfer, owing to the larger mass of the transferred fragment. The cluster is transferred at high excitation energy (tens of $\mathrm{MeV}$ ) as can be expected based on optimum $Q$-value considerations. Table I provides some characteristics of the reaction studied in this paper for both $\mathrm{t}$ and $\alpha$ transfers to ${ }^{98} \mathrm{Rb}$.
TABLE I. Relevant parameters for both the $\mathrm{t}$ - and the $\alpha$-transfer channels on ${ }^{98} \mathrm{Rb}$ : The table provides the $Q$ value for the transfer to the ground state $\left(Q_{\mathrm{gg}}\right)$, the expected optimum $Q$ value $\left(Q_{\mathrm{opt}}\right)$ calculated at leading order according to Ref. [41], and the corresponding excitation energy $\left(E_{\mathrm{opt}}^{*}\right)$ at which the cross section is thought to be highest. The last row refers to the $\mathrm{t}-{ }^{98} \mathrm{Rb}$ and $\alpha-{ }^{98} \mathrm{Rb}$ separation energies (S.E.).

\begin{tabular}{lcc}
\hline \hline$(\mathrm{MeV})$ & $\mathrm{t}$ transfer & $\alpha$ transfer \\
\hline$Q_{\mathrm{gg}}$ & 13.6 & 7.6 \\
$Q_{\mathrm{opt}}$ & -5.1 & -10.4 \\
$E_{\mathrm{opt}}^{*}$ & 18.7 & 18 \\
S.E. & 16 & 10 \\
\hline \hline
\end{tabular}

As can be seen from Table I, these channels are characterized by large positive $Q_{\mathrm{gg}}$ and negative $Q_{\mathrm{opt}}$ values, which result in high excitation energies peaked where the cross section is expected to be the highest. $Q_{\text {opt }}$ was calculated according to Ref. [41] using the first-order approximation expression. These energies are larger than the energy threshold for fragment $-{ }^{98} \mathrm{Rb}$ separation but still well below the fission barrier such that the final nucleus deexcites initially by neutron evaporation and, subsequently, by $\gamma$ emission as will be shown below. Furthermore, the simple structure of ${ }^{7} \mathrm{Li}$ ensures that few degrees of freedom are involved in the process so that the main part of the flux in the outgoing transfer channels is in cluster transfer.

In this section, the potential for exploiting this mechanism with radioactive beams in inverse kinematics in order to study neutron-rich nuclei is presented. The reaction dynamics will be discussed in the framework of a binary process as a direct transfer of a clusterlike particle to weakly-bound states located close to the continuum using a DWBA approximation.

Figure 4 presents $\gamma$ spectra Doppler corrected on an event-by-event basis obtained in coincidence with $\alpha$ and $\mathrm{t}$ particles. These correspond to $\mathrm{t}$ and $\alpha$ transfers on ${ }^{98} \mathrm{Rb}$ and populate ${ }^{101} \mathrm{Sr}^{*}$ and ${ }^{102} \mathrm{Y}^{*}$, respectively, followed by neutron evaporation. Since the reaction took place on ${ }^{98} \mathrm{Sr}$ as well, ${ }^{101} \mathrm{Y}^{*}$ and ${ }^{102} \mathrm{Zr}^{*}$ are populated, and transitions from products after neutron evaporation are also identified and are labeled appropriately (Fig. 4). Note that the detection of the cluster in coincidence with $\gamma$ rays resulted in a clean trigger on the final channel of interest. Several nuclei were identified: they correspond to different numbers of evaporated neutrons depending on the excitation energy of the final systems above the neutron threshold. It is worth noting that a typical neutron separation energy for nuclei in this mass region is between 5 and $6 \mathrm{MeV}$, whereas the excitation energy of the final systems as measured in this work is between 11 and $23 \mathrm{MeV}$. For reactions on ${ }^{98} \mathrm{Rb}$, both $3 n$ and $2 n$ channels are observed, whereas in the case of ${ }^{98} \mathrm{Sr}$, only the $2 n$ channel is present for both $\mathrm{t}$ and $\alpha$ transfers. By comparing the excitation energy in the projectilelike systems following transfer with the respective neutron separation energies $\left(S_{\mathrm{xn}}\right)$, the difference between reactions on the ${ }^{98} \mathrm{Rb}$ and ${ }^{98} \mathrm{Sr}$ beam components in terms of observed final residues can be understood. In fact, in the case of ${ }^{98} \mathrm{Rb}$, the average excitation energy of the ${ }^{101} \mathrm{Sr}$ final system is $19 \mathrm{MeV}$. With $S_{3 n}\left({ }^{101} \mathrm{Sr}\right)=13.292 \mathrm{MeV}$, 


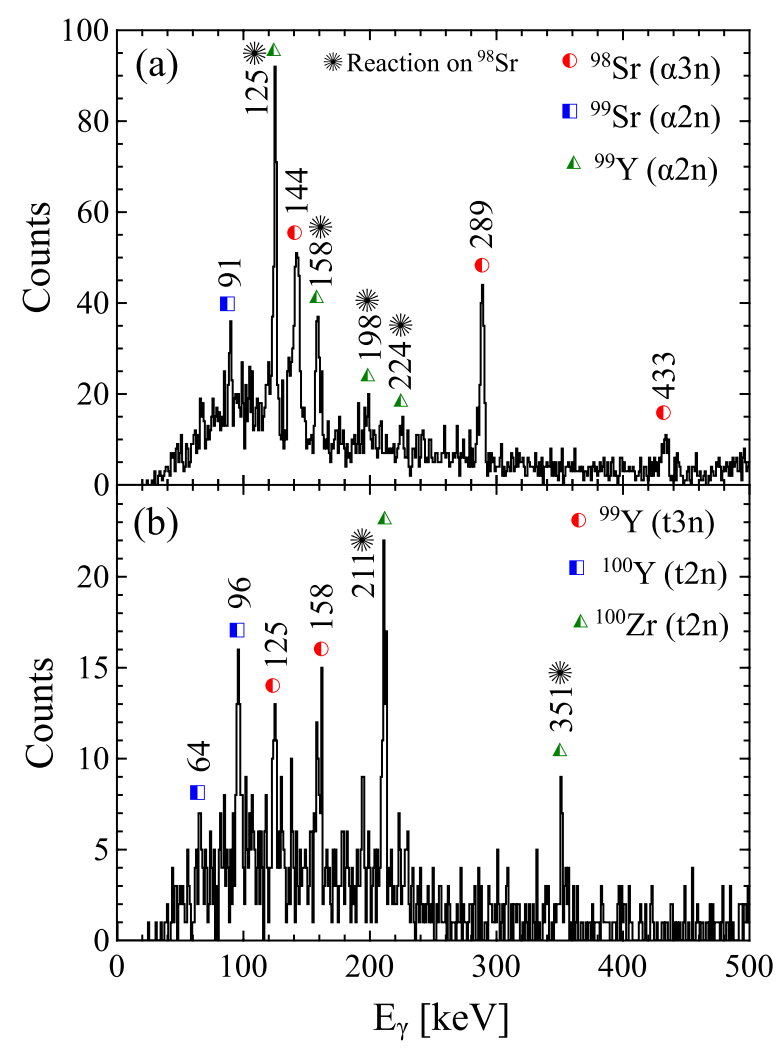

FIG. 4. (Color online) Particle-gated $\gamma$-ray spectra for clustertransfer channels followed by neutron evaporation for reactions on both ${ }^{98} \mathrm{Rb}$ and ${ }^{98} \mathrm{Sr}$. (a) $\alpha-\gamma$ coincidence data corresponding to the t-transfer channel. For reactions on ${ }^{98} \mathrm{Rb}$, the $3 n$ channel $\left({ }^{98} \mathrm{Sr}\right)$ is indicated by circles (red), whereas the $2 n$ one $\left({ }^{99} \mathrm{Sr}\right.$ ) is identified by squares (blue). Transitions corresponding to reactions on ${ }^{98} \mathrm{Sr}$ are labeled with a star with the $2 n$ channel $\left({ }^{99} \mathrm{Y}\right)$ marked by triangles (green). (b) t $-\gamma$ coincidence spectra corresponding to the $\alpha$-transfer channel. For reactions on ${ }^{98} \mathrm{Rb}$, the $3 n$ channel $\left({ }^{99} \mathrm{Y}\right)$ is indicated by circles (red) whereas the $2 n$ one $\left({ }^{100} \mathrm{Y}\right)$ is identified by squares (blue). Transitions corresponding to reactions on ${ }^{98} \mathrm{Sr}$ are labeled with a star with the $2 n$ channel $\left({ }^{100} \mathrm{Zr}\right.$ ) marked by triangles (green).

this accounts for the evaporation of up to three neutrons. In contrast, for ${ }^{98} \mathrm{Sr}$, the average excitation energy in ${ }^{101} \mathrm{Y}$ is $16 \mathrm{MeV}$ and $\left.S_{3 n}{ }^{101} \mathrm{Y}\right)=16.674 \mathrm{MeV}$. This justifies the absence of the $3 n$ channel in this case.

Despite the high excitation energy and high angular momentum of the systems after transfer, yrast states were observed with spins up to $6 \hbar$ only in the case of $t$ transfer and up to $4 \hbar$ for $\alpha$ transfer. This is mainly due to the limitation imposed by the low intensity of the beam as well as by the beam composition, resulting in insufficient statistics to observe $\gamma$ rays from higher-spin and off-yrast levels. The average expected spins can also be deduced from the measured distribution of neutron-evaporation channels. The difference in the observed distribution of residues was investigated by comparing the measured channel yields with the results of calculations with the statistical model CASCADE [39]. For this purpose, the decay of the final nuclei was studied as a function of entry spin, considering the final systems at their highest measured excitation energies. The results are presented in

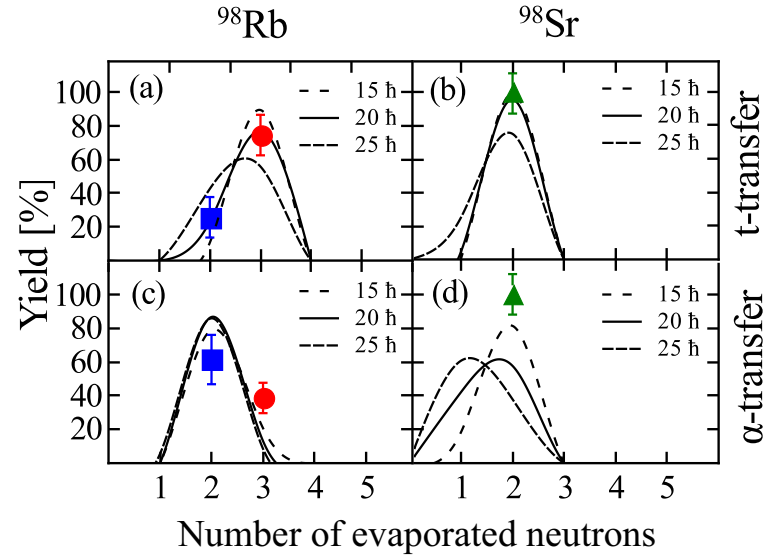

FIG. 5. (Color online) Experimental yield of neutron evaporation for both $\mathrm{t}$ and $\alpha$ transfers on the ${ }^{98} \mathrm{Rb}$ and ${ }^{98} \mathrm{Sr}$ beam components compared with CASCADE predictions for different spin distributions. Panels (a) and (b) show the experimental data corresponding to $t$ transfer whereas panels (c) and (d) correspond to $\alpha$-transfer channels (see text for details). Squares (blue) and triangle (green) symbols correspond to $2 n$ evaporation whereas dots (red) refer to $3 n$ channels.

Fig. 5 where it is seen that the data are reproduced well by assuming a spin of $20 \hbar$ for $\mathrm{t}$ transfer, whereas $15 \hbar$ is required to fit $\alpha$ transfer. This observation supports the expectation that states with medium-high energy and medium-high spin are populated in $\mathrm{t}$ and $\alpha$ cluster-transfer processes. Furthermore, an analysis of the results of the CASCADE calculations indicates that, in the case of $\mathrm{t}$ transfer on ${ }^{98} \mathrm{Rb}$, an average excitation energy of $6 \mathrm{MeV}$ and an average angular momentum of $16 \hbar$ are predicted for the $2 n$ channel, whereas $2 \mathrm{MeV}$ and $9.5 \hbar$ are expected for the $3 n$ one. These characteristics indicate that low-lying states with moderate-to-high spin in exotic nuclei produced in cluster-transfer processes induced by radioactive beams should be available for extensive structure studies with $\gamma$-ray spectroscopy techniques.

In order to make more detailed predictions, a better understanding of the reaction dynamics for cluster-transfer processes involving neutron-rich nuclei is desirable.

In the present paper, the cluster-transfer mechanism was studied for the ${ }^{98} \mathrm{Rb}+{ }^{7} \mathrm{Li}$ system through comparisons with the results of calculations with the code FRESCO [28]. (Note that calculations for ${ }^{98} \mathrm{Sr}+{ }^{7} \mathrm{Li}$ give similar results [42]). To do so, the elastic cross section was first investigated in order to obtain the optical parameters of the scattering potential in the initial mass partition. Global optical parameters for ${ }^{7} \mathrm{Li}$ elastic scattering were used [43], resulting in a good fit of the experimental data as demonstrated in the inset of Fig. 6 where the ratio between the cross sections for elastic and Rutherford scatterings is presented. As can be seen, the experimental angular distribution is consistent with essentially pure Rutherford scattering for nearly all the measured angles, except perhaps for the largest ones where interference from the nuclear potential is expected to affect the data. A scaling of the experimental elastic data to the theoretical distribution provided the normalization factor required to obtain absolute experimental cross sections. 


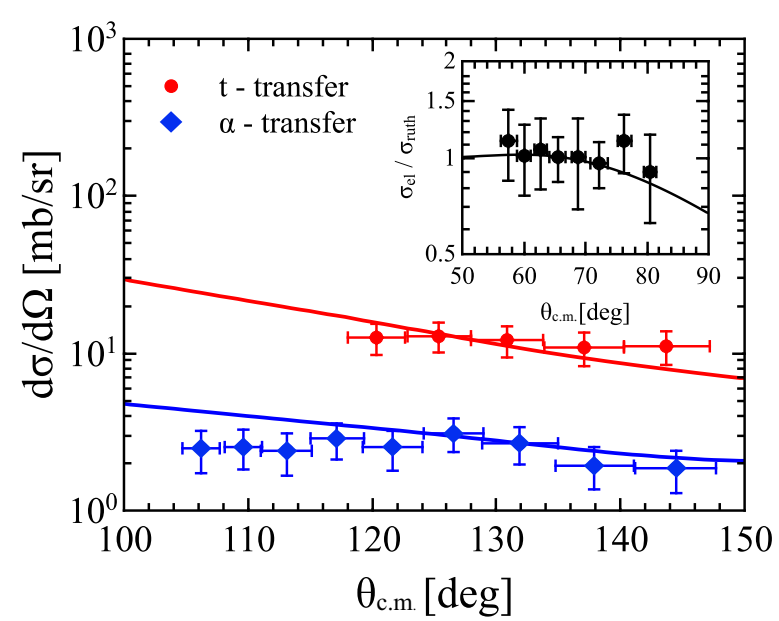

FIG. 6. (Color online) Angular distribution for $\mathrm{t}$ [dots (red)] and $\alpha$ [diamonds (blue)] transfers on ${ }^{98} \mathrm{Rb}$ compared with theoretical calculations (solid lines) as explained in the text. Data were rescaled with the same normalization factor. Inset: ratio between the elastic and the Rutherford cross sections as a function of the ${ }^{7} \mathrm{Li}$ scattering angle. Data were fitted in order to obtain the optical parameters for elastic scattering.

The transfer cross section data were interpreted by considering the reaction as a binary process involving the direct transfer of a clusterlike particle. The scattering potentials in the final mass partitions were chosen to fit the present experimental data, starting from optical-model parameters for heavy-ion scattering described in Ref. [33]. These parameters are presented in Table II. The ${ }^{7} \mathrm{Li}$ ground-state wave function was described in the framework of a cluster model, considering an $\alpha(\mathrm{t})$ core in the case of $\mathrm{t}(\alpha)$ transfer [44], resulting in the $\mathrm{t}$ and $\alpha$ particles being in a relative $P$ state. Furthermore, a Gaussian interaction was used as a binding mean-field potential $V_{\alpha-\mathrm{t}}$ [45]. The final states accessed by the transfer were also described within a cluster model using a standard Woods-Saxon potential. Since the transfer populates states above the $\alpha-{ }^{98} \mathrm{Rb}$ and $\mathrm{t}-{ }^{98} \mathrm{Rb}$ separation energies, the final levels were treated within a weakly-bound approximation in order to take into account their real position in the continuum. As a matter of fact, their wave functions were constructed such that a very small binding energy was reproduced and the dependence of the cross sections on the angular momentum and the number of nodes was investigated. Finally, the scattering wave functions were calculated using a one-step DWBA approach in the so-called post representation of the interaction $\mathcal{V}_{\text {int }}$ without considering the remnant terms: $\mathcal{V}_{\text {int }}^{\text {post }} \approx V_{\alpha-\mathrm{t}}$.
Figure 6 displays the angular distributions for $t$ and $\alpha$ transfers where experimental data were rescaled with a common factor obtained from the elastic-scattering data.

To test the ability of model calculations to reproduce the difference in relative cross sections between the two transfer channels, theoretical distributions obtained with the FRESCO code are also given in the figure after normalization to the data with a single normalization coefficient. It can be seen that the ratio between the two experimental distributions is reproduced well by theoretical calculations, provided that: (i) states up to $\ell_{\max }=3$ and $\ell_{\max }=5$ are included for $\mathrm{t}$ and $\alpha$ transfers, respectively, and that (ii) the number of nodes in the case of the $\alpha$ transfer is twice that in the t-transfer channel. The choice of the angular momenta was driven by the fact that the cross sections exhibit a maximum at $\ell_{\max }$ for both $\mathrm{t}$ and $\alpha$ transfers. Several states, in steps of $0.5 \mathrm{MeV}$, were considered for each $\ell$ value up to $\ell_{\max }$. Notably, no dependence of the shape of the angular distributions on the angular momentum transferred was observed. The differential cross sections as a function of the excitation energy of the final systems are presented in Fig. 7 for both $\mathrm{t}$ transfer [panel (a)] and $\alpha$ transfer [panel (b)]. The experimental distributions were reconstructed, assuming two-body kinematics, by deducing the excitation energies from the angles and the kinetic energy of the detected $t$ and $\alpha$ particles. First, it may be noticed that both distributions exhibit a clear maximum at the energy deduced from the optimum $Q$ value. This is in line with expectations based on semiclassical considerations which predict a pronounced peak for the cross section at high excitation energy. Moreover, the theoretical calculations performed in this paper can reproduce the shape of the experimental distributions (Fig. 7). In the case of $t$ transfer, the cross-section behavior is reproduced better than in the case of $\alpha$ transfer: This is consistent with the weakly-bound approximation as the peak of the former distribution is close to the $t-{ }^{98} \mathrm{Rb}$ separation energy, making such an approximation reliable. On the other hand, the $\alpha$-transfer distribution is peaked at $8 \mathrm{MeV}$ above the $\alpha-{ }^{98} \mathrm{Rb}$ separation energy, rendering the weakly-bound assumption less suitable. Also, in this case, it should be noted that the angular momentum of the final states has no impact on the peak position. These results demonstrate that the transfer can be qualitatively described as a direct process and that such an approach is able to reproduce the main properties of the reaction. Nevertheless, to achieve a more quantitative description, the coupling to other channels should be included, together with a more realistic representation of the states in the continuum with a proper discretization of the phase space.

TABLE II. Woods-Saxon optical-model parameters for the incoming channel $\left({ }^{7} \mathrm{Li}+{ }^{98} \mathrm{Rb}\right)$ and the outgoing t- and $\alpha$-transfer channels $\left(\alpha+{ }^{101} \mathrm{Sr}\right.$ and $\left.\mathrm{t}+{ }^{102} \mathrm{Y}\right)$. The former are adopted from Ref. [43] and reproduce the elastic scattering well, whereas the latter were obtained by fitting the present experimental data.

\begin{tabular}{lcccccr}
\hline \hline Channel & $V(\mathrm{MeV})$ & $R_{V}(\mathrm{fm})$ & $a_{V}(\mathrm{fm})$ & $W(\mathrm{MeV})$ & $r_{W}(\mathrm{fm})$ & $a_{W}(\mathrm{fm})$ \\
\hline${ }^{7} \mathrm{Li}+{ }^{98} \mathrm{Rb}$ & 114.2 & 1.286 & 0.853 & 15.643 & 1.739 & 0.809 \\
$\alpha+{ }^{101} \mathrm{Sr}$ & 140.0 & 1.200 & 1.200 & 10.0 & 1.200 & 1.200 \\
$\mathrm{t}+{ }^{102} \mathrm{Y}$ & 80.0 & 1.250 & 1.500 & 10.0 & 1.250 & 1.500 \\
\hline \hline
\end{tabular}




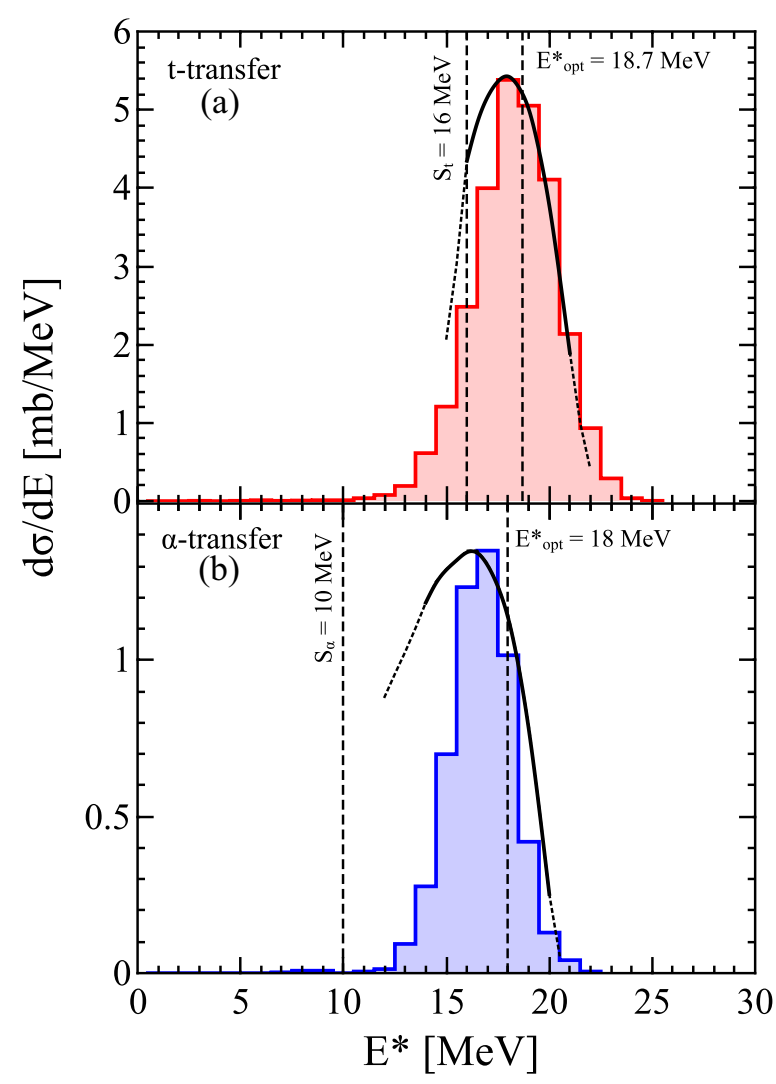

FIG. 7. (Color online) Top panel: differential cross section as a function of excitation energy for the t-transfer channel on ${ }^{98} \mathrm{Rb}$. The colored area corresponds to the experimental distribution whereas theoretical calculations are shown as a solid line (see text for details). The $t$ separation energy is also indicated $\left(S_{\mathrm{t}}=16 \mathrm{MeV}\right)$ as well as the expected maximum excitation energy $\left(E_{\mathrm{opt}}^{*}=18.7 \mathrm{MeV}\right)$ deduced from the optimum $Q$ value. Bottom panel: differential cross section as a function of excitation energy for the $\alpha$-transfer channel on ${ }^{98} \mathrm{Rb}$. The colored area corresponds to the experimental distribution whereas theoretical calculations are shown as a solid line (see text for details). The $\alpha$ separation energy is also indicated $\left(S_{\alpha}=10 \mathrm{MeV}\right)$ as well as the expected maximum excitation energy $\left(E_{\mathrm{opt}}^{*}=18 \mathrm{MeV}\right)$ deduced from the optimum $Q$ value.

For the sake of completeness, the absolute cross sections were obtained for both $\mathrm{t}$ and $\alpha$ transfers $\left(\sigma_{\mathrm{t}}\right.$ and $\left.\sigma_{\alpha}\right)$ by normalizing the data with the scaling factor extracted from elastic scattering (see above) and integrating the experimental data over the same solid angle $(\approx 10 \%$ of $4 \pi)$ :

$$
\begin{aligned}
\sigma_{\mathrm{t}} & =26.6 \pm 0.7 \mathrm{mb} \\
\sigma_{\alpha} & =5.3 \pm 0.2 \mathrm{mb}
\end{aligned}
$$

Inclusive cross sections cannot be extracted since the angular distributions are limited by the experimental setup. Nevertheless, the values above are comparable in magnitude with those of previous experiments performed in direct kinematics where values on the order of 100 and $10 \mathrm{mb}$ were measured for $t$ and $\alpha$ transfers, respectively [21].
The possibility that part of the measured $\alpha$ and t particles, interpreted here as originating from direct $\mathrm{t}$ - and $\alpha$-transfer processes, may come from other reactions, such as incomplete fusion, cannot be entirely ruled out. It has recently been shown in a direct kinematics experiment [46] that the measured cross sections can be reproduced well by considering a two-step process where ${ }^{7} \mathrm{Li}$ breakup is followed by the fusion of either fragment. Although it is impossible to disentangle such two-step processes from the direct reactions used above, the satisfactory description achieved here argues in favor of the fact that other reaction mechanisms either only impact the absolute scale of the cross sections or only contribute little for systems and energies, such as those considered here.

\section{CONCLUSIONS}

In this work, an exploratory study of the ${ }^{98} \mathrm{Rb}+{ }^{7} \mathrm{Li}$ reaction at the Coulomb barrier energy $(2.85 \mathrm{MeV} /$ nucleon) was performed at REX-ISOLDE to investigate cluster-transfer processes with radioactive beams in inverse kinematics. The potential of using such cluster-transfer processes to access yrast and near-yrast states in neutron-rich nuclei was verified. The results, based on particle- $\gamma$ coincidences, demonstrate the suitability of the experimental method to populate mediumhigh energy and medium-high spin states with yields sufficient for $\gamma$-ray spectroscopic studies, even with radioactive beams of relatively low intensity. Furthermore, the reaction dynamics was studied, and comparisons with theoretical calculations achieve a satisfactory description of the process in terms of a direct transfer. Further experimental information is needed to better understand the process, especially in terms of inclusive cross sections.

The experimental technique examined here can be readily exploited to investigate nuclear structure in even more exotic regions of the nuclide chart, once new radioactive beam facilities, such as HIE-ISOLDE, SPIRAL2, SPES, ISAC, FRIB, etc., come on line with beams of higher energy and higher intensity.

\section{ACKNOWLEDGMENTS}

This work was funded by Istituto Nazionale di Fisica Nucleare (INFN-Italy), by FWO-Vlaanderen (Belgium), by Grant No. GOA/2010/010 (BOF KU Leuven), by the Interuniversity Attraction Poles Programme initiated by the Belgian Science Policy Office (BriX network P7/12), by the Polish Ministry of Science and Higher Education under Contract No. N-N202-263238, by the European Commission within the Seventh Framework Programme through I3-ENSAR (Contract No. RII3-CT-2010-262010), by the German BMBF (Contracts No. 06K-167, No. 06KY205I, No. 05P09PKCI5, and No. 05P12PKFNE), by the US Department of Energy, Office of Science, Office of Nuclear Physics under Contract No. DEAC02-06CH11357, and by the European Commission through the Marie Curie Actions Contract No. PIEFGA-2011-30096. 
[1] T. Otsuka, Phys. Scr. T 152, 014007 (2013).

[2] R. Kanungo, Phys. Scr. T 152, 014002 (2013).

[3] A. Gade, Phys. Scr. T 152, 014004 (2013).

[4] R. V. F. Janssens, Phys. Scr. T 152, 014005 (2013).

[5] L. W. Iskra et al., Phys. Rev. C 89, 044324 (2014).

[6] B. Fornal et al., Phys. Rev. Lett. 87, 212501 (2001).

[7] B. Fornal et al., Phys. Rev. C 67, 034318 (2003).

[8] R. Broda, J. Phys. G: Nucl. Part. Phys. 32, R151 (2006).

[9] L. Corradi, G. Pollarolo, and S. Szilner, J. Phys. G: Nucl. Part. Phys. 36, 113101 (2009).

[10] G. Benzoni et al., Eur. Phys. J. A 45, 287 (2010).

[11] R. Broda, K. H. Maier, B. Fornal, J. Wrzesiński, B. Szpak, M. P. Carpenter, R. V. F. Janssens, W. Królas, T. Pawłat, and S. Zhu, Phys. Rev. C 84, 014330 (2011).

[12] B. Szpak et al., Phys. Rev. C 83, 064315 (2011).

[13] D. Montanari et al., Phys. Lett. B 697, 288 (2011).

[14] D. Montanari et al., Phys. Rev. C 84, 054613 (2011).

[15] D. Montanari et al., Phys. Rev. C 85, 044301 (2012).

[16] S. Bottoni et al., Phys. Rev. C 85, 064621 (2012).

[17] N. Cieplicka et al., Phys. Rev. C 86, 054322 (2012).

[18] G. D. Dracoulis, A. P. Byrne, T. Kibédi, T. R. McGoram, and S. M. Mullins, J. Phys. G: Nucl. Part. Phys. 23, 1191 (1997).

[19] S. M. Mullins, G. D. Dracoulis, A. P. Byrne, T. R. McGoram, S. Bayer, R. A. Bark, R. T. Newman, W. A. Seale, and F. G. Kondev, Phys. Rev. C 61, 044315 (2000).

[20] A. Jungclaus et al., Phys. Rev. C 67, 034302 (2003).

[21] R. M. Clark et al., Phys. Rev. C 72, 054605 (2005).

[22] D. S. Judson et al., Phys. Rev. C 76, 054306 (2007).

[23] H. Watanabe et al., Phys. Rev. C 79, 024306 (2009).

[24] D. R. Tilley, C. M. Cheves, J. L. Godwin, G. M. Hale, H. M. Hofmann, J. H. Kelley, C. G. Sheu, and H. R. Weller, Nucl. Phys. A 708, 3 (2002).

[25] P. E. Hodgson and E. Betak, Phys. Rep. 374, 1 (2003).
[26] O. Kester et al., Nucl. Instrum. Methods Phys. Res., Sect. B 204, 20 (2003).

[27] P. Van Duppen and K. Riisager, J. Phys. G: Nucl. Part. Phys. 38, 024005 (2011).

[28] I. J. Thompson, Comput. Phys. Rep. 7, 167 (1988).

[29] F. Wenander, J. Instrum. 5, C10004 (2010).

[30] N. Warr et al., Eur. Phys. J. A 49, 40 (2013).

[31] V. Bildstein et al., Eur. Phys. J. A 48, 85 (2012).

[32] A. N. Ostrowski, S. Cherubini, T. Davinson, D. Groombridge, A. M. Laird, A. Musumarra, A. Ninane, A. di Pietro, A. C. Shotter, and P. J. Woods, Nucl. Instrum. Methods Phys. Res., Sect. A 480, 448 (2002).

[33] R. A. Broglia and A. Winther, Heavy Ion Reactions: Parts 1 and 2 (Frontiers in Physics) (Addison-Wesley, Reading, MA 1991).

[34] L. F. Canto, P. R. S. Gomes, R. Donangelo, and M. S. Hussein, Phys. Rep. 424, 1 (2006).

[35] http://www.nndc.bnl.gov.

[36] C. M. Folden et al., Phys. Rev. C 79, 064318 (2009).

[37] E. Clement (private communication).

[38] D. H. Luong, M. Dasgupta, D. J. Hinde, R. du Rietz, R. Rafiei, C. J. Lin, M. Evers, and A. Diaz-Torres, Phys. Lett. B 695, 105 (2011)

[39] F. Puhlhofer, Nucl. Phys. A 280, 267 (1977).

[40] J. Wilczynski, Nucl. Phys. A 216, 386 (1973).

[41] W. Henning, Y. Eisen, H. J. Körner, D. G. Kovar, J. P. Schiffer, S. Vigdor, and B. Zeidman, Phys. Rev. C 17, 2245(R) (1978).

[42] S. Bottoni, Ph.D. thesis, Università degli Studi di Milano (Italy) and KU Leuven (Belgium) (2015).

[43] J. Cook, Nucl. Phys. A 388, 153 (1982).

[44] B. Buck and A. A. Pilt, Nucl. Phys. A 280, 133 (1977).

[45] B. Buck and A. C. Merchant, J. Phys. G: Nucl. Part. Phys. 14, 211 (1988).

[46] A. Shrivastava et al., Phys. Lett. B 718, 931 (2013). 